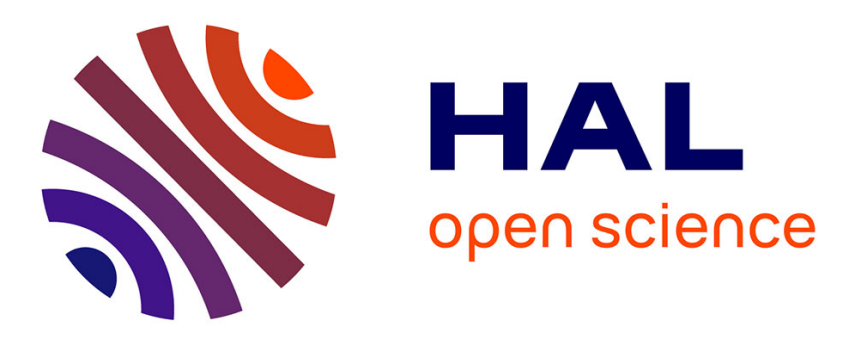

\title{
Learning motor coordination under resistive viscous force fields at the joint level with an upper-limb robotic exoskeleton
}

\author{
Tommaso Proietti, Agnès Roby-Brami, Nathanael Jarrasse
}

\section{- To cite this version:}

Tommaso Proietti, Agnès Roby-Brami, Nathanael Jarrasse. Learning motor coordination under resistive viscous force fields at the joint level with an upper-limb robotic exoskeleton. Converging Clinical and Engineering Research on Neurorehabilitation II, pp.1175-1179, 2017. hal-02110208

\section{HAL Id: hal-02110208 \\ https://hal.science/hal-02110208}

Submitted on 25 Apr 2019

HAL is a multi-disciplinary open access archive for the deposit and dissemination of scientific research documents, whether they are published or not. The documents may come from teaching and research institutions in France or abroad, or from public or private research centers.
L'archive ouverte pluridisciplinaire HAL, est destinée au dépôt et à la diffusion de documents scientifiques de niveau recherche, publiés ou non, émanant des établissements d'enseignement et de recherche français ou étrangers, des laboratoires publics ou privés. 


\title{
Learning motor coordination under resistive viscous force fields at the joint level with an upper-limb robotic exoskeleton
}

\author{
Tommaso Proietti, Agnès Roby-Brami, and Nathanaël Jarrassé
}

\begin{abstract}
In the field of rehabilitation robotics, few researchers have been focusing on the problem of controlling motor coordination in post-stroke patients. Studies on coordination learning, when the robotic devices act at the joint level on multiple interaction points, as in the case of exoskeletons, are lacking. For this reason, we studied on $\mathbf{1 0}$ healthy subjects the possibility of learning a non-natural inter-joint coordination while performing a pointing task. This coordination was induced by a 4-DOF robotic exoskeleton, applying resistive force fields at the joint level. Preliminary results showed the capability of our controller to modify human healthy natural coordination after exposition to the fields and generalization of these effects to movements which were never exposed to these constraints.
\end{abstract}

\section{INTRODUCTION}

Restoring motor coordination control in post-stroke patients is a central objective for modern neurorehabilitation. Stroke survivors suffer from stereotyped unnatural patterns of movements, occurring during any upper-limb motion, which can encourage negative compensations and potentially limit motor recovery [1]. Despite the diffusion of robotic exoskeletons [2] and their natural capability to perform jointlevel control, most of the existing controllers for rehabilitation robotics are not focused on restoring natural inter-joint coordination [3], [4]. We used a controller, the Kinematic Synergy Controller (KSC) [5], to impose an alternative motor coordination on healthy subjects while performing Pointing Tasks (PT) in a 3D space, through joint-level resistive force fields. The objective was to observe two potential different phenomena at the motor control level: adaptation to the fields i.e. the existence of after-effects modifying the human natural joint coordination, and generalization or transfer of these effects on PT that were never exposed to any force field. We know that adaptation and generalization may occur when subjects are exposed to force fields produced by planar robots [6], but only Mistry et al. [7] showed preliminary results of adaptation, due to exposition to force fields acting at the joint level, using an exoskeleton, thus directly applying the fields along the upper-limb and not only at the end-point.

\section{MATERIALS AND METHODS}

All the experiments were conducted with an ABLE exoskeleton, a 4-DOF lightweight robotic device made by CEA-LIST [8]. Ten subjects were asked to perform PT, sitting on a stool in front of a 7-DOF WAM manipulator

Authors are with Sorbonne Universités, UPMC Univ. Paris 06, CNRS, UMR 7222, the Institute of Intelligent Systems and Robotics (ISIR), 4 place Jussieu, 75005, Paris, France. Agnès Roby-Brami is also with U1150, Institut National de la Santé et de la Recherche Médicale (INSERM), France. \{proietti, roby-brami, jarrasse\}@isir.upmc.fr
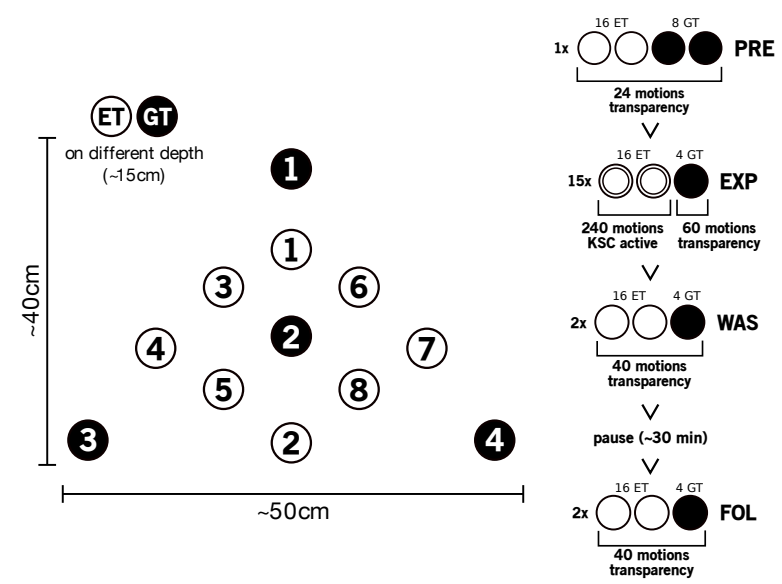

Fig. 1. Experimental protocol. On the left, Experimental target ET and Generalization Target GT; on the right, the four phases of the experiment.

(c) Barrett Technology), on the top of which a push button was mounted. Starting from a resting position (the upperarm along the body, with the elbow bent about 90 degrees and the forearm along the leg), the subjects were asked to push the button on the WAM through a plastic rod, screwed on a commercial wrist guard for avoiding wrist motion. The task lasted less than $4 \mathrm{~s}$ and the starting time of every motion was manually triggered by the users through a button in the other hand. Once the WAM button was pushed, the exoskeleton was passively bringing back the upper-limb towards the starting position. The subjects were free to move from the starting position to the button, that is they were not asked to follow any specific trajectory. The WAM moved through 12 different final positions, grouped in Experimental Targets (ET, 8 positions) and in Generalization Targets (GT, 4 positions), shown in figure 1 . These positions were fixed for all the subject. The average total time for each experiment was about 2 hours. Initially, every subject was set inside the exoskeleton for few minutes to practice free movements in transparency (ABLE only gravity compensated). After this initial training, the experiment consisted of 4 phases, figure 1: preliminary (PRE), experiment (EXP), wash-out (WAS), and follow up (FOL). For each phase, the subject was asked to point the different positions within the robotic exoskeleton. In particular the phases were:

- PRE (24 total PT) 2x8 ET followed by 2x4 GT,

- EXP (300 total PT) 15 repetitions of 2x8 ET plus 4 GT, thus 20 PT per repetition,

- WAS (40 total PT) two repetitions of 2x8 ET + $4 \mathrm{GT}$,

- FOL (40 total PT) two repetitions of $2 \times 8 \mathrm{ET}+4 \mathrm{GT}$. 


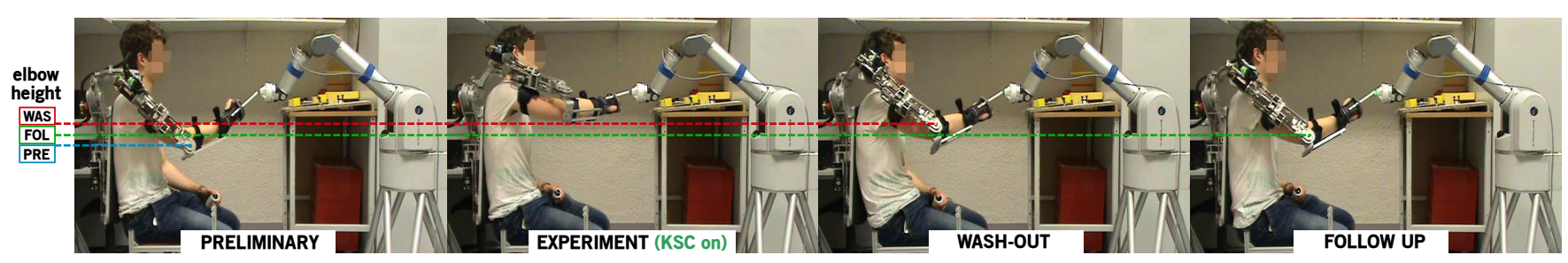

Fig. 2. Final elbow height for ET position \#3. These snapshots show an example of adaptation to the resistive force fields: in the first picture (PRE) the subject is performing naturally. When the KSC is active, the subject final posture is clearly deviated from the natural pose (EXP). In the last two pictures (respectively WAS and FOL), the effect on the elbow height once removed the force fields: the final postures are in between the natural coordination and the one forced by the KSC.

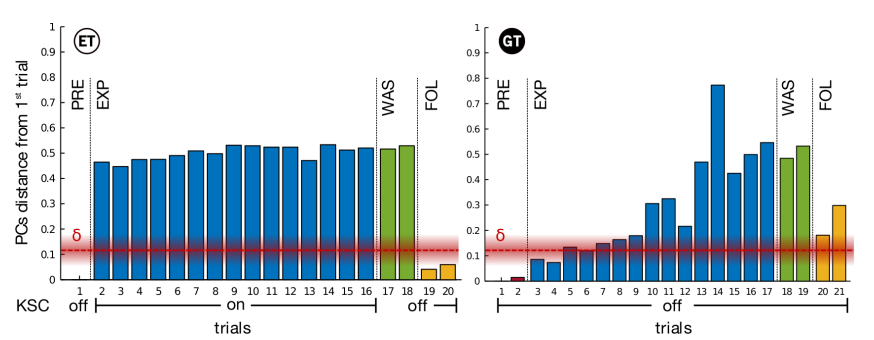

Fig. 3. PCs distance from first trial in ET pointing task (left plot) and in GT pointing task (right one) for one typical subject. The phases are shown in different colors (red PRE, blue EXP, green WAS, yellow FOL), while the baseline of stable coordination $\delta \pm S T D$ is dashed in red.

All the sequences of pointing tasks were performed by blocks of $8 \mathrm{ET}$ or $4 \mathrm{GT}$ trials presented in the same randomized order for each subject. PRE, EXP, and WAS were performed in sequence, while before FOL there was a pause of about 30 minutes, in which the subject rested, detached from the exoskeleton. Finally, the KSC was active only during the pointing tasks towards ET and only during EXP. Otherwise, the robot was set in transparency. Therefore, as shown in figure 1, the KSC was resisting to the subject free motion only on 240 motions over the 404 total motion of each experiment. This fact means that GT movements were always unconstrained natural motions.

\section{PRELIMINARY RESULTS}

An example of the after-effects, which were visibly occurring during the experiment, is shown in fig. 2. In particular, this sequence of pictures shows, for the same ET, the final upper-limb position. Clearly the subject adapted to an interjoint coordination in between the natural coordination (PRE) and the one forced by the KSC (EXP). These effects always occurred during the experiment (10 subjects out of 10). Furthermore, we found that 8 subjects showed different final joint postures even in the GT pointing task, thus generalizing what was learnt during the pointing under resistive force fields towards ET (GT were never exposed to resistance by ABLE). By performing Principal Component Analysis (PCA) on the joint velocity and considering the distance between the Principal Components (PCs) subspaces, we determined the differences in dynamical coordination, i.e. the joint trajectory modifications, not only at the final posture but also during the gesture. We performed baseline tests on 5 healthy subjects doing motions with the robot in transparency and we took the distance between PCs $(\delta=0.11 \pm 0.05)$ as a reference for stable coordination. When pointing at ET in 6 subjects we observed an effect of adaptation when comparing the initial coordination with the motions in WAS (left plot of fig. 3), i.e. a larger value than the reference $\delta$. When pointing at GT, half of the subjects produced increasing trends when comparing to the initial natural PCs, meaning that trial-by-trial generalization of the post-effects was occurring (right plot of fig. 3). These facts show that adaptation and generalization did not concern only the static final posture, but appeared dynamically during the whole execution of the PT, and that post-effects were still effective 30 minutes later the end of the training.

\section{PERSPECTIVES}

While upper-limb exoskeletons are starting to being used for clinical rehabilitation, there is still a need to understand how subjects, even healthy, are adapting and learning motor behaviours within these devices. This preliminary work illustrates part of the observable phenomena. However deeper analysis and quantification of these results is necessary by taking into consideration other motor control variables, such as, for example, smoothness and end-effector trajectories, but also by evaluating different force fields and tasks, like more difficult and involving path tracking instead of simpler pointing task.

\section{REFERENCES}

[1] N. Jarrassé, T. Proietti, V. Crocher, J. Robertson, A. Sahbani, G. Morel, and A. Roby-Brami, Robotic exoskeletons: a perspective for the rehabilitation of arm coordination in stroke patients, Front Hum Neurosci, 2014, vol. 8.

[2] P. Maciejasz, J. Eschweiler, K. Gerlach-Hahn, A. Jansen-Troy, and S. Leonhardt, A survey on robotic devices for upper limb rehabilitation, JNER, 2014.

[3] T. Proietti, V. Crocher, A. Roby-Brami, and N. Jarrassé, Upperlimb robotic exoskeletons for neurorehabilitation: a review on control strategies, RBME, 2016.

[4] N. Nordin, S. Xie, and B. Wünsche, Assessment of movement quality in robot-assisted upper limb rehabilitation after stroke: a review, JNER, 2014.

[5] V. Crocher, A. Sahbani, and G. Morel, Constraining upper limb synergies of hemiparetic patients using a robotic exoskeleton in the perspective of neuro-rehabilitation, TNSRE, 2012, vol. 20:3.

[6] R. Shadmehr, and F. Mussa-Ivaldi, Adaptive representation of dynamics during learning of a motor task, The Journal of Neuroscience, 1994, vol. 14:5

[7] M. Mistry, P. Mohajerian, and S. Schaal, Arm movement experiments with joint space force fields using an exoskeleton robot, ICORR, 2005.

[8] P. Garrec, J.P. Friconneau, Y. Measson, and Y. Perrot, ABLE an innovative transparent exoskeleton for the upper-limb, IROS, 2008. 